\title{
Effect of Pore Packing Defects in 2-D Ordered Mesoporous Carbons on Ionic Transport
}

\author{
Da-Wei Wang ${ }^{a}$, Feng Li ${ }^{a}$, Hai-Tao Fang ${ }^{a, b}$, Min Liu ${ }^{a}$, Gao-Qing Lu ${ }^{c}$, Hui-Ming Cheng ${ }^{a *}$
}

a Shenyang National Laboratory for Materials Science, Institute of Metal Research, Chinese Academy of Sciences, 72 Wenhua Road, Shenyang 110016, China

b Materials Science and Engineering School, Harbin Institute of Technology, 92 West Dazhi Street, Harbin 150001, China

c Australian Research Council Centre for Functional Nanomaterials, School of Engineering, The University of Queensland, QLD 4072, Australia

A two-electrode system was assembled as follows. Sandwich-type electrodes were prepared by pressing the OMC powders (c.a. $5.5 \mathrm{mg}$ ) between two sponge-like nickel disks (with diameter $15 \mathrm{~mm}$ ) under $2 \mathrm{MPa}$ in a stainless steel cylinder. Before electrochemical measurements, the electrodes were vacuum impregnated for 12 hours to guarantee that the electrolyte ions could penetrate thoroughly into the mesopores. Two electrodes were separated by a nylon paper and assembled in a beaker-type cell to form a supercapacitor. The electrolyte

* Corresponding Author: Professor Hui-Ming Cheng

E-mail: cheng@imr.ac.cn Fax: 86-24-2390-3126 
was a $6 \mathrm{M} \mathrm{KOH}$ aqueous solution. The cyclic voltammogram were collected on Solartron 1287/1260 electrochemical systems. The voltage scanning range for CV was 0 to $1 \mathrm{~V}$.

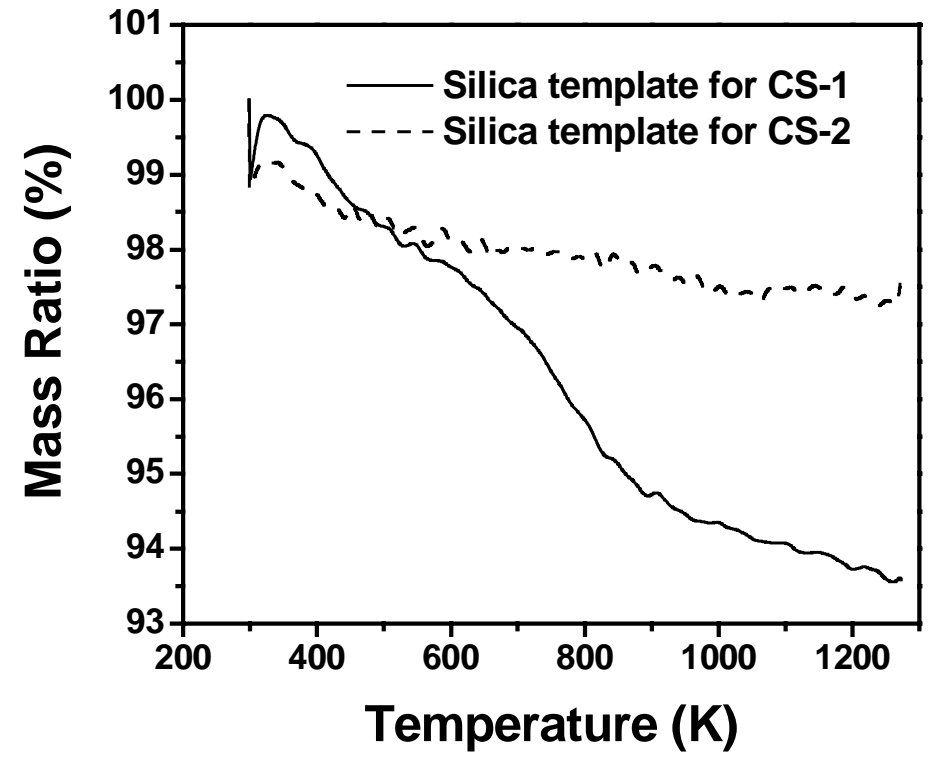

Figure $1 \mathrm{~S}$ TG curves for silica templates in air atmosphere at heating rate of $10 \mathrm{~K} / \mathrm{min}$.

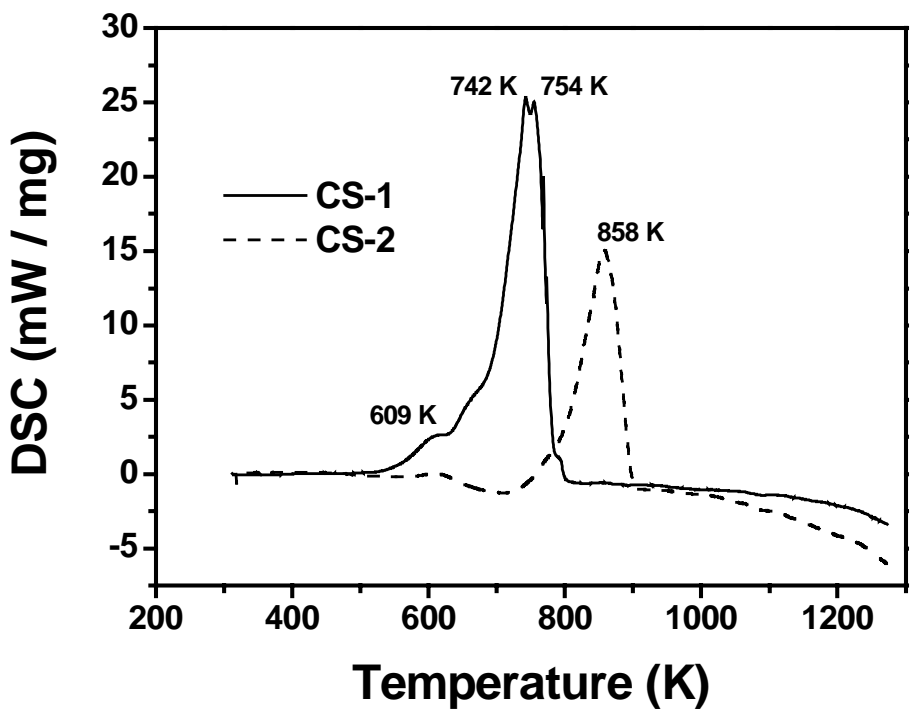

Figure 2S DSC curves for CS-1 and CS-2 in air atmosphere at heating rate of $10 \mathrm{~K} / \mathrm{min}$. 
Table 1S The gravimetric capacitance of CS-1 and CS-2 under different measurement conditions with a two-electrode system

\begin{tabular}{|c|c|c|c|c|c|c|c|}
\hline \multirow{2}{*}{$\begin{array}{l}\text { Capacitor } \\
\text { Type }\end{array}$} & \multirow[t]{2}{*}{ Sample } & \multicolumn{6}{|c|}{ Gravimetric capacitance at different voltage scan rates (F/g) } \\
\hline & & $5 \mathrm{mV} / \mathrm{s}$ & $10 \mathrm{mV} / \mathrm{s}$ & $20 \mathrm{mV} / \mathrm{s}$ & $30 \mathrm{mV} / \mathrm{s}$ & $40 \mathrm{mV} / \mathrm{s}$ & $50 \mathrm{mV} / \mathrm{s}$ \\
\hline \multirow[t]{2}{*}{ 2-Electrode } & CS-1 & 147.9 & 139.2 & 132.3 & 126.2 & 121.1 & 115.4 \\
\hline & CS-2 & 124.9 & 122.1 & 117.5 & 114.2 & 107.9 & 105.9 \\
\hline
\end{tabular}

Table $2 \mathrm{~S}$ The specific capacitance per unit surface area of CS-1 and CS-2 under different measurement conditions with a two-electrode system

\begin{tabular}{|c|c|c|c|c|c|c|c|}
\hline \multirow{2}{*}{$\begin{array}{l}\text { Capacitor } \\
\text { Type }\end{array}$} & \multirow[t]{2}{*}{ Sample } & \multirow{2}{*}{$\begin{array}{l}\begin{array}{l}\text { Specific } \\
\left(\mathrm{F} / \mathrm{m}^{2}\right)^{\mathrm{a}}\end{array} \\
5 \mathrm{mV} / \mathrm{s}\end{array}$} & \multicolumn{2}{|c|}{ surface capacitance } & at differen & voltage & scan rates \\
\hline & & & $10 \mathrm{mV} / \mathrm{s}$ & $20 \mathrm{mV} / \mathrm{s}$ & $30 \mathrm{mV} / \mathrm{s}$ & $40 \mathrm{mV} / \mathrm{s}$ & $50 \mathrm{mV} / \mathrm{s}$ \\
\hline \multirow[t]{2}{*}{ 2-Electrode } & CS-1 & 0.136 & 0.128 & 0.122 & 0.116 & 0.111 & 0.106 \\
\hline & $\mathrm{CS}-2$ & 0.102 & 0.1 & 0.096 & 0.093 & 0.088 & 0.087 \\
\hline
\end{tabular}

a The Capacitance per unit surface area $\left(\mathrm{C}_{\mathrm{s}}\right)$ is calculated by equation $\mathrm{C}_{\mathrm{s}}=\mathrm{C}_{\mathrm{g}} / \mathrm{S}_{\mathrm{BET}}$, where $\mathrm{C}_{\mathrm{g}}$ is the gravimetric capacitance listed in Table $1 \mathrm{~S}, \mathrm{~S}_{\mathrm{BET}}$ is the BET specific surface area. 